\title{
ZERO TRAINING FOR BCI - REALITY FOR BCI SYSTEMS BASED ON EVENT-RELATED POTENTIALS
}

\author{
Michael Tangermann ${ }^{1}$, Pieter-Jan Kindermans ${ }^{3}$, Martijn Schreuder ${ }^{2}$, \\ Benjamin Schrauwen ${ }^{3}$, Klaus-Robert Müller ${ }^{2}$ \\ ${ }^{1}$ Excellence Cluster BrainLinks-BrainTools, University of Freiburg, Germany \\ ${ }^{2}$ Dept. Machine Learning, BBCI Group, Technical University Berlin, Germany \\ ${ }^{3}$ Electronics and Information Systems Department, Ghent University, Belgium \\ mt@we-are.de
}

\begin{abstract}
This contribution reviews how usability in BrainComputer Interfaces (BCI) can be enhanced. As an example, an unsupervised signal processing approach is presented, which tackles usability by an algorithmic improvement from the field of machine learning. The approach completely omits the necessity of a calibration recording for BCIs based on event-related potential (ERP) paradigms. The positive effect is twofold - first, the experimental time is shortened and the productive online use of the BCI system starts as early as possible. Second, the unsupervised session avoids the usual paradigmatic break between calibration phase and online phase, which is known to introduce data-analytic problems related to non-stationarity.
\end{abstract}

Keywords: Brain-Computer Interface, Machine Learning, Unsupervised Classification, Event-Related Potentials, Spatial Auditory Attention, Usability

\section{Introduction}

Usability challenges in Brain-Computer Interfaces so far have impeded their application in every-day scenarios. Recently, this problem has been recognized and addressed by a number of research projects, which have taken the field closer to a true usability for patients. Among them hybrid BCI paradigms [1, 2], simplified user interfaces [3], dry electrode systems $[4,5]$, reduced electrode sets $[6,7]$, transfer learning approaches $[8,9]$, shared control principles [10], improvements in BCI paradigms [11, 12] and stimulus modalities $[13,14,15]$ need to be recognized.

\section{Methods}

Now, another brick has been placed to form this important fundament - a completely unsupervised signal processing approach [16]. Discarding calibration recordings completely - even for novel subjects - this method allows for a kick-start usage of BCI systems, which are based on eventrelated potentials (ERP) like the P300.

Making use of an established six-class auditory spelling paradigm [12], examples of the online experience with this Bayesian unsupervised approach are presented, and a comparison to the traditional approach involving supervised LDA classification and explicit calibration recordings is made.

\section{Results}

The positive effect of the unsupervised classification approach for ERPs is twofold. First, the overall experimental time is shortened by the unsupervised approach, as the productive online use of the BCI system starts as early, as enough evidence has been collected from the data. Second, the unsupervised session is monolithic in the sense, that it does not undergo any paradigmatic change and the feedback mode remains the same throughout the session. This strategy avoids the usual paradigmatic break between calibration phase and online phase, which is known to introduce data-analytic problems related to the increasing nonstationarity of the data.

\section{Acknowledgement}

The authors are thankfor for funding by the BOFGOA Project Home-MATE (Ghent University Special Research Fund), by the Bernstein Focus Neurotechnology (01GQ0850) and by the DFG (MU987/14-1).

\section{Bibliography}

[1] J. d. R. Millán, R. Rupp, G. Müller-Putz, R. MurraySmith, C. Giugliemma, M. Tangermann, C. Vidaurre, F. Cincotti, A. Kübler, R. Leeb, et al., "Combining brain-computer interfaces and assistive technologies: state-of-the-art and challenges," Frontiers in neuroscience, vol. 4, 2010.

[2] G. R. Müller-Putz, C. Breitwieser, F. Cincotti, R. Leeb, M. Schreuder, F. Leotta, M. Tavella, L. Bianchi, A. Kreilinger, A. Ramsay, et al., "Tools for brain-computer interaction: a general concept for a hybrid BCI," Frontiers in neuroinformatics, vol. 5, 2011.

[3] T. Kaufmann, S. Völker, L. Gunesch, and A. Kübler, "Spelling is just a click away-a user-centered braincomputer interface including auto-calibration and predictive text entry," Frontiers in Neuroscience, vol. 6, 2012.

[4] F. Popescu, S. Fazli, Y. Badower, B. Blankertz, and K.-R. Müller, "Single trial classification of motor 
imagination using 6 dry EEG electrodes," PloS one, vol. 2, no. 7, p. e637, 2007.

[5] T. O. Zander, M. Lehne, K. Ihme, S. Jatzev, J. Correia, C. Kothe, B. Picht, and F. Nijboer, "A dry EEG-system for scientific research and brain-computer interfaces," Frontiers in neuroscience, vol. 5, 2011.

[6] C. Sannelli, C. Vidaurre, K.-R. Müller, and B. Blankertz, "CSP patches: an ensemble of optimized spatial filters. an evaluation study," Journal of Neural Engineering, vol. 8, no. 2, p. 025012, 2011.

[7] T. N. Lal, M. Schröder, T. Hinterberger, J. Weston, M. Bogdan, N. Birbaumer, and B. Schölkopf, "Support vector channel selection in BCI," Biomedical Engineering, IEEE Transactions on, vol. 51, no. 6, pp. 1003-1010, 2004.

[8] M. Krauledat, M. Tangermann, B. Blankertz, and K.R. Müller, "Towards zero training for brain-computer interfacing," PLoS One, vol. 3, no. 8, p. e2967, 2008.

[9] S. Fazli, C. Grozea, M. Danoczy, B. Blankertz, F. Popescu, and K.-R. Müller, "Subject independent EEG-based BCI decoding," Advances in Neural Information Processing Systems, vol. 22, pp. 513-521, 2009.

[10] F. Galán, M. Nuttin, E. Lew, P. W. Ferrez, G. Vanacker, J. Philips, and J. d. R. Millán, "A brainactuated wheelchair: asynchronous and non-invasive brain-computer interfaces for continuous control of robots," Clinical Neurophysiology, vol. 119, no. 9, pp. 2159-2169, 2008.

[11] V. V. Nikulin, F. U. Hohlefeld, A. M. Jacobs, and G. Curio, "Quasi-movements: A novel motorcognitive phenomenon," Neuropsychologia, vol. 46, no. 2, pp. 727-742, 2008.

[12] M. Schreuder, T. Rost, and M. Tangermann, "Listen, you are writing! speeding up online spelling with a dynamic auditory BCI," Frontiers in Neuroscience, vol. 5, 2011.

[13] S. Schaeff, M. S. Treder, B. Venthur, and B. Blankertz, "Exploring motion VEPs for gaze-independent communication," Journal of Neural Engineering, vol. 9, no. 4, p. 045006, 2012.

[14] M. Tangermann, M. Schreuder, S. Dähne, J. Höhne, S. Regler, A. Ramsay, M. Quek, J. Williamson, and R. Murray-Smith, "Optimized stimulation events for a visual ERP BCI," Int J Bioelectromagnetism Volume, vol. 13, no. 3, pp. 119-120, 2011.

[15] J. Höhne, K. Krenzlin, S. Dähne, and M. Tangermann, "Natural stimuli improve auditory BCIs with respect to ergonomics and performance," Journal of Neural Engineering, vol. 9, no. 4, p. 045003, 2012.
[16] P.-J. Kindermans, D. Verstraeten, and B. Schrauwen, "A bayesian model for exploiting application constraints to enable unsupervised training of a P300based BCI," PloS one, vol. 7, no. 4, p. e33758, 2012. 